\title{
Differential equations and dispersion relations for the sunrise amplitudes
}

\author{
Lorenzo Tancredi* \\ Institute for Theoretical Particle Physics, KIT, 76128 Karlsruhe, Germany \\ E-mail: lorenzo.tancredi@kit.edu
}

\section{Ettore Remiddi}

DIFA, Universitá di Bologna and INFN, Sezione di Bologna, I-40126 Bologna, Italy

E-mail: ettore.remiddiabo.infn.it

\begin{abstract}
The study of the imaginary part and of the corresponding dispersion relations of Feynman graph amplitudes within the differential equations method can provide a powerful tool for the solution of the equations, especially in the massive case. We illustrate the main features of the approach by applying it to the case of two-loop massive sunrise graph and to the two-loop kite integral with three internal massive propagators. We will then show how to derive compact analytical results for the first two non-trivial orders of their $\varepsilon=(4-d) / 2$ expansion and to continue them analytically for all physical values of the incoming momentum.
\end{abstract}

Loops and Legs in Quantum Field Theory

24-29 April 2016

Leipzig, Germany

${ }^{*}$ Speaker. 


\section{Introduction}

The calculation of higher order corrections in the Standard Model has become even more important after the start of the LHC experimental program. Practically, this requires the computation of complicated massless and massive multi-loop and/or multi-leg Feynman integrals. If until recently the calculation of two-loop corrections to most $2 \rightarrow 2$ processes relevant for LHC phenomenology was considered an outstanding task, the situation has changed dramatically in the last years, thanks to the development of diverse computational techniques. A prominent role in this has been played by the discovery of integration-by-parts identities (IBPs) [1], the method of differential equations [2-4], the introduction of the multiple polylogarithms (MPLs) [5-10], the definition of a so-called canonical basis of master integrals [11], and the use of the Magnus exponentiation [12]. These techniques seem to be particularly well suited as long as we limit ourselves to massless calculations. On the other hand, starting at two-loops, the increase in the number of internal masses (together, of course, with the increase of scales in the problem), brings us very soon outside the realm of MPL functions, forcing us to re-think our approach to the calculation of Feynman integrals [13-21]. The problem seems to be strictly intertwined with the apparent impossibility of finding a basis of masters integrals, whose differential equations decouple in the limit $d \rightarrow 4$ or, equivalently, $d \rightarrow 2 n$ with $n \in \mathbb{N}$ [22]. We recall here also that algorithms for studying arbitrarily complicated systems of differential equations - but limited for the moment to the univariate case have been proposed in [23] and in a more general framework in [24, 25].

In a recent paper the authors have shown that the study of the imaginary parts and related dispersion relations satisfied by the Feynman amplitudes, within the differential equation frame, can provide another useful practical tool for their evaluation, in the massless as well as in the massive case [26]. The imaginary parts of Feynman graphs can be obtained by the Cutkosky-Veltman rule [27-29] or, more interestingly, one can try to compute it through the differential equations that they satisfy. Often, in fact, their solution becomes substantially simpler when restricted to the imaginary part only. In what follows we will illustrate the main points of the method and its application to the calculation of the two-loop massive sunrise and the two-loop kite integral. We refer to the original paper for details [26].

\section{Combining differential equations and dispersion relations}

Let us consider two Feynman amplitudes, say $A(d ; u)$ and $B(d ; u)$, both functions of the dimensions $d$ and of a given external invariant $u$. Let us assume, for simplicity, that $A(d ; u)$ is simpler than $B(d ; u)$, namely that $A(d ; u)$ can be obtained from $B(d ; u)$ by pinching one or more propagators. As it is well known, we expect in this case that the differential equations for $B(d ; u)$ will contain $A(d ; u)$ as inhomogeneous term, i.e. schematically

$$
\frac{d}{d u} B(d ; u)=h(d ; u) B(d ; u)+g(d ; u) A(d ; u)+\text { other subtopologies. }
$$

Now it is clear that, once the imaginary part of $A(d ; u)$, say $\operatorname{Im} A(d ; u)$, is obtained, one has at disposal the dispersive representation for $A(d ; u)$, namely an expression of the form

$$
A(d ; u)=\frac{1}{\pi} \int d t \operatorname{Im} A(d ; t) \frac{1}{t-u}
$$


(where the limit of integration have been skipped for ease of typing). Such a representation turns out to be very useful when the amplitude $A(d ; u)$ appears within the inhomogeneous terms of some other differential equation, regardless of the actual analytical expression of $A(d ; u)$, see (2.1). Indeed, as the whole dependence on $u$ is in the denominator $(t-u)$, one can work out its contribution by considering only that denominator. By inserting (2.2) into (2.1) one finds at once

$$
\frac{d}{d u} B(d ; u)=h(d ; u) B(d ; u)+\frac{1}{\pi} \int d t \operatorname{Im} A(d ; t) \frac{g(d ; u)}{t-u}+\text { other subtopologies. }
$$

such that the $t$-integration can be considered as, so to say, frozen, until the dependence on the variable $u$ (the variable of the differential equation) has been properly processed.

The importance of formula (2.3) should be self-evident. Suppose that we can compute the amplitude $A(d ; u)$ and that the latter evaluates to functions outside the realm of MPLs (for example, integrals over elliptic integrals). If this is the case, the approach outlined here allows us to confine the complexity of $A(d ; u)$ in the $t$-dependent integral only and to integrate the differential equation for $B(d ; u)$ considering only the denominator $(t-u)$. In particular, if nor the homogeneous solution of (2.3) neither the remaining subtopologies introduce further functions beyond the MPLs, the integration over $(t-u)$ will necessarily produce MPLs only ${ }^{1}$. As a first application of the procedure outlined here we considered the two-loop massive sunrise and the kite integral, which are relevant for the computation of the two-loop corrections to the electron self-energy in QED.

\section{The kite integral family}

Let us start defining the Feynman graph associated to the kite integral

$$
\begin{aligned}
\mathscr{I}\left(n_{1}, n_{2}, n_{3}, n_{4}, n_{5}\right) & =\rightarrow \\
& =\int \mathfrak{D}^{d} k \mathfrak{D}^{d} l \frac{1}{D_{1}^{n_{1}} D_{2}^{n_{2}} D_{3}^{n_{3}} D_{4}^{n_{4}} D_{5}^{n_{5}}}
\end{aligned}
$$

where two propagators are massless and two are massive, as follows

$$
\begin{array}{ll}
D_{1}=k^{2}+m^{2}, & D_{2}=l^{2}, \quad D_{3}=(k-l)^{2}+m^{2}, \\
D_{4}=(k-p)^{2}, & D_{5}=(l-p)^{2}+m^{2},
\end{array}
$$

with $-p^{2}=s$ and $p^{2}>0$ when $p$ is spacelike. We put $m=1$ and define $u=s / m^{2}$. Using IBPs we find 8 independent master integrals which we choose as follows

$$
\begin{array}{ll}
M_{1}(d ; u)=\mathscr{I}(2,0,2,0,0), & M_{2}(d ; u)=\mathscr{I}(2,0,2,1,0), \\
M_{3}(d ; u)=\mathscr{I}(0,2,2,1,0), & M_{4}(d ; u)=\mathscr{I}(0,2,1,2,0), \\
M_{5}(d ; u)=\mathscr{I}(2,1,0,1,2), & M_{6}(d ; u)=\mathscr{I}(1,0,1,0,1), \\
M_{7}(d ; u)=\mathscr{I}(2,0,1,0,1), & M_{8}(d ; u)=\mathscr{I}(1,1,1,1,1) .
\end{array}
$$

\footnotetext{
${ }^{1}$ Here and in the following whenever we say MPLs, what we really have in mind is any function obtained by iterative integrations over d-log forms only.
} 
$M_{1}, \ldots, M_{5}$ can be written in terms of HPLs only and we will not consider them anymore here, referring to [26] for their explicit expressions. The remaining three integrals, $M_{6}, M_{7}$ and $M_{8}$, are instead the two master integrals of the two-loop sunrise graph (i.e. $M_{6}$ and $\left.M_{7}\right)$ and of the kite $\left(M_{8}\right)$ and are more complicated. In order to simplify their integration, we choose a canonical basis for the simple subtopologies as follows

$$
\begin{aligned}
& f_{1}(d ; u)=4(d-4)^{2} M_{1}(d ; u), \quad f_{2}(d ; u)=(d-4)^{2} u M_{2}(d ; u), \\
& f_{3}(d ; u)=(d-4)^{2} u M_{3}(d ; u), \quad f_{4}(d ; u)=(d-4)^{2}(1-u)\left[\frac{1}{2} M_{3}(d ; u)+M_{4}(d ; u)\right], \\
& f_{5}(d ; u)=(d-4)^{2} u^{2} M_{5}(d ; u) .
\end{aligned}
$$

Moreover, using general considerations based on the form of the imaginary part of the sunrise amplitude for $d \approx 4$ and $d \approx 2$, together with Tarasov-Lee dimensional shifting identities [30,31], we build a basis for $M_{6}, M_{7}, M_{8}$ which is particular well suited for their integration as Laurent series in $(d-4)$, see $[22,26]$ for details. The basis reads

$$
\begin{aligned}
& h_{6}(d ; u)=g_{6}(d-2 ; u), \\
& h_{7}(d ; u)=g_{7}(d-2 ; u), \\
& f_{8}(d ; u)=(d-4)^{3}(d-3) u M_{8}(d ; u),
\end{aligned}
$$

where

$$
\begin{aligned}
g_{6}(d ; u) & =f_{6}(d ; u), \\
g_{7}(d ; u) & =-2(d-4)^{2}(u-1)(u-9) M_{7}(d ; u)+\frac{1}{3}\left(u^{2}-6 u+21\right) f_{6}(d ; u) \\
& +6(d-2)(d-4)^{2}(u-1) M_{6}(d ; u) .
\end{aligned}
$$

We stress here that in (3.5), $g_{6}(d ; u)$ and $g_{7}(d ; u)$ are taken in $d-2$ space-time dimensions.

\subsection{The two-loop massive sunrise subtopologies}

With the basis (3.5), the differential equations satisfied by the two master integrals of the sunrise graph read

$$
\frac{d}{d u}\left(\begin{array}{l}
h_{6}(d ; u) \\
h_{7}(d ; u)
\end{array}\right)=B(u)\left(\begin{array}{l}
h_{6}(d ; u) \\
h_{7}(d ; u)
\end{array}\right)+(d-4) D(u)\left(\begin{array}{c}
h_{6}(d ; u) \\
h_{7}(d ; u)
\end{array}\right)+\left(\begin{array}{l}
0 \\
1
\end{array}\right),
$$

where the two matrices $B(u), D(u)$ are defined as

$$
\begin{gathered}
B(u)=\frac{1}{6 u(u-1)(u-9)}\left(\begin{array}{cc}
3\left(3+14 u-u^{2}\right) & -9 \\
(u+3)\left(3+75 u-15 u^{2}+u^{3}\right) & -3\left(3+14 u-u^{2}\right)
\end{array}\right) \\
D(u)=\frac{1}{6 u(u-9)(u-1)}\left(\begin{array}{c}
6 u(u-1) \\
(u+3)\left(9+63 u-9 u^{2}+u^{3}\right) 3(u+1)(u-9)
\end{array}\right) .
\end{gathered}
$$


Note that the differential equations have regular singular points in $u=0, u=1, u=9$ and $u=$ $\pm \infty$. We are interested in the solution of (3.7) as Laurent series in $(d-4)$, in the four relevant regions $-\infty<u<0,0<u<1,1<u<9$ and $9<u<\infty$. To this aim, we need to first solve the homogeneous system for $d=4$, i.e. to find a set of two independent solutions, say $\left(I_{1}(u), I_{2}(u)\right)$ and $\left(J_{1}(u), J_{2}(u)\right)$, such that the matrix of the solutions

$$
G(u)=\left(\begin{array}{ll}
I_{1}(u) & J_{1}(u) \\
I_{2}(u) & J_{2}(u)
\end{array}\right) \quad \text { satisfies } \quad \frac{d}{d u} G(u)=B(u) G(u) .
$$

Note that since $\operatorname{Tr}(B(u))=0$ the Wronskian of the four solutions, $W(u)=I_{1}(u) J_{2}(u)-I_{2}(u) J_{1}(u)$, must be independent of $u$, i.e.

$$
\frac{d}{d u} W(u)=\frac{d}{d u} \operatorname{det}(G(u))=\operatorname{Tr}(B(u)) \operatorname{det}(G(u))=0 .
$$

Euler's method of the variation of constants allows to write the general solution of a coupled system of linear differential equations, provided that one has at disposal its homogeneous solution. Since no general algorithm is known to solve the homogeneous system, we must resort to some physical arguments to be able to proceed. We do this by noticing that, by taking the imaginary part of (3.7), and setting $d=4$, we are left with

$$
\frac{d}{d u}\left(\begin{array}{c}
\operatorname{Im} h_{6}(4 ; u) \\
\operatorname{Im} h_{7}(4 ; u)
\end{array}\right)=B(u)\left(\begin{array}{c}
\operatorname{Im} h_{6}(4 ; u) \\
\operatorname{Im} h_{7}(4 ; u)
\end{array}\right)
$$

which implies that the four dimensional imaginary parts of the two master integrals $h_{6}$ and $h_{7}$ solve the homogeneous system. The sunrise develops an imaginary part for $u>9$, which can be readily computed using Cutkosky-Veltman rules [27-29]. We get

$$
\begin{aligned}
& \frac{1}{\pi} \operatorname{Im} h_{6}(4 ; u)=I(0, u) \\
& \frac{1}{\pi} \operatorname{Im} h_{7}(4 ; u)=\frac{1}{(u-1)(u-9)}\left[\frac{u^{2}-6 u+21}{6} I(0, u)-\frac{1}{2} I(2, u)\right],
\end{aligned}
$$

where the functions $I(n, u)$ are defined as

$$
I(n, u)=\int_{4}^{(\sqrt{u}-1)^{2}} d b \frac{b^{n}}{\sqrt{R_{4}(b, u)}}
$$

and $R_{4}(d, u)$ is the fourth-order polynomial

$$
R_{4}(b, u)=b(b-4)\left((\sqrt{u}-1)^{2}-b\right)\left((\sqrt{u}+1)^{2}-b\right) .
$$

As showed explicitly in $[14,26]$, these integrals are not all linearly independent, but instead, using integration-by-parts, one can prove that there are only two master integrals, which we choose as $I(0, u)$ and $I(2, u)$. The latter can be related by an elementary change of variables to the complete 
elliptic integrals of first and second kind [14]. Using (3.13) it is straightforward to prove that they fulfil Eqs. (3.10), such that, for $9<u<\infty$, we can define a first homogeneous solution as

$$
I_{1}^{(9, \infty)}(u)=I(0, u), \quad I_{2}^{(9, \infty)}(u)=I(2, u) .
$$

Taking inspiration from the solution above, one can build up a second pair of independent functions as follows

$$
J_{1}^{(9, \infty)}(u)=\int_{0}^{4} d b \frac{1}{\sqrt{-R_{4}(b, u)}}, \quad J_{2}^{(9, \infty)}(u)=\int_{0}^{4} d b \frac{b^{2}}{\sqrt{-R_{4}(b, u)}}+\frac{\pi}{3}(u+3),
$$

where it is a simple exercise to verify that

$$
\frac{d}{d u}\left(\begin{array}{l}
J_{1}^{(9, \infty)}(u) \\
J_{2}^{(9, \infty)}(u)
\end{array}\right)=B(u)\left(\begin{array}{c}
J_{1}^{(9, \infty)}(u) \\
J_{2}^{(9, \infty)}(u)
\end{array}\right)
$$

as required. Summarising we have found two pairs of independent real valued solutions, valid in the range $9<u<\infty$, such that their matrix

$$
G^{(9, \infty)}(u)=\left(\begin{array}{l}
I_{1}^{(9, \infty)}(u) J_{1}^{(9, \infty)}(u) \\
I_{2}^{(9, \infty)}(u) J_{2}^{(9, \infty)}(u)
\end{array}\right) \quad \text { fulfils } \quad \frac{d}{d u} G^{(9, \infty)}(u)=B(u) G^{(9, \infty)}(u)
$$

In ref. [26] similar solution matrices are constructed in all remaining regions, $-\infty<u<0$, $0<u<1$ and $1<u<9$, and the analytic continuation from one region to the other is explicitly worked out. In order to indicate the matrix of solution in a generic region $a<u<b$, we use the notation $G^{(a, b)}(u)$. With the normalization given above, in every region $(a, b)$ the Wronskian of the solutions is independent of $u$, as expected, and can be computed to be

$$
I_{1}^{(a, b)}(u) J_{2}^{(a, b)}(u)-I_{2}^{(a, b)}(u) J_{1}^{(a, b)}(u)=\pi,
$$

which is nothing but the Legendre relation among complete elliptic integrals of first and second kind. We are now ready to use Euler's method of the variation of constants in order to write the complete solution of the system Eq.(3.7). In what follows we will drop the superscripts $(a, b)$ from all formulas for simplicity, writing for instance $G(u)$ instead of $G^{(9, \infty)}(u)$, since the manipulations do not depend on those. We will restore the dependence once we specialize the solution in any given region. We start performing the rotation

$$
\left(\begin{array}{c}
h_{6}(d ; u) \\
h_{7}(d ; u)
\end{array}\right)=G(u)\left(\begin{array}{c}
m_{6}(d ; u) \\
m_{7}(d ; u)
\end{array}\right)
$$

such that the new functions $m_{6}(d ; u)$ and $m_{7}(d ; u)$ fulfil the equations

$$
\frac{d}{d u}\left(\begin{array}{c}
m_{6}(d ; u) \\
m_{7}(d ; u)
\end{array}\right)=(d-4) \frac{1}{\pi} M(u)\left(\begin{array}{c}
m_{6}(d ; u) \\
m_{7}(d ; u)
\end{array}\right)+\frac{1}{\pi}\left(\begin{array}{c}
-J_{1}(u) \\
I_{1}(u)
\end{array}\right)
$$

with

$$
M(u)=\pi G^{-1}(u) D(u) G(u), \quad \text { and } \quad G^{-1}(u)=\frac{1}{\pi}\left(\begin{array}{cc}
J_{2}(u) & -J_{1}(u) \\
-I_{2}(u) & I_{1}(u)
\end{array}\right) .
$$


Using the condition on the Wronskian (3.20), one can show that

$$
I_{1}(u) \frac{d J_{1}(u)}{d u}-J_{1}(u) \frac{d I_{1}(u)}{d u}=-\frac{3 \pi}{2} \frac{1}{u(u-1)(u-9)},
$$

which allows to simplify the matrix $M(u)$ and write is as a total differential. Its entries read

$$
\begin{aligned}
& M_{11}(u)=-\frac{d}{d u}\left(\frac{(u+3)^{2}}{6} I_{1}(u) J_{1}(u)\right)+\frac{\pi}{4}\left(\frac{2}{u-9}+\frac{2}{u-1}-\frac{1}{u}\right), \\
& M_{12}(u)=-\frac{d}{d u}\left(\frac{(u+3)^{2}}{6} I_{1}(u) I_{1}(u)\right), \\
& M_{21}(u)=+\frac{d}{d u}\left(\frac{(u+3)^{2}}{6} J_{1}(u) J_{1}(u)\right), \\
& M_{22}(u)=+\frac{d}{d u}\left(\frac{(u+3)^{2}}{6} I_{1}(u) J_{1}(u)\right)+\frac{\pi}{4}\left(\frac{2}{u-9}+\frac{2}{u-1}-\frac{1}{u}\right) .
\end{aligned}
$$

Written in this form, the iterative structure of the solution in powers of $(d-4)$ becomes manifest. Eqs. (3.22) with the matrix (3.25) are now ready to be expanded in Laurent series in $(d-4)$, integrated and matched to proper boundary conditions. We skip all mathematical details here and report the final solution of the integration. We expand the master integrals as follows

$$
h_{j}(d ; u)=\sum_{\alpha=0}^{\infty}(d-4)^{\alpha} h_{j}^{(\alpha)}(u), \quad \text { for } \quad j=6,7,
$$

such that the first non-zero order is $(d-4)^{0}$, and the solution for the master integrals, valid in the region $0<u<1$, reads

$$
\begin{aligned}
& h_{6}^{(0)}(u)=\frac{1}{\pi}\left[J_{1}^{(0,1)}(u) \int_{0}^{u} d t I_{1}^{(0,1)}(t)-I_{1}^{(0,1)}(u)\left(\int_{0}^{u} d t J_{1}^{(0,1)}(t)-\mathrm{Cl}_{2}\left(\frac{\pi}{3}\right)\right)\right], \\
& h_{7}^{(0)}(u)=\frac{1}{\pi}\left[J_{2}^{(0,1)}(u) \int_{0}^{u} d t I_{1}^{(0,1)}(t)-I_{2}^{(0,1)}(u)\left(\int_{0}^{u} d t J_{1}^{(0,1)}(t)-\mathrm{Cl}_{2}\left(\frac{\pi}{3}\right)\right)\right],
\end{aligned}
$$

where the Clausen function is defined as

$$
\mathrm{Cl}_{2}(x)=-\int_{0}^{x} \ln \left|2 \sin \frac{y}{2}\right| d y=\frac{i}{2}\left(\operatorname{Li}_{2}\left(e^{-i x}\right)-\operatorname{Li}_{2}\left(e^{i x}\right)\right) .
$$

By analytically continuing the solution above threshold, i.e. for $u>9$, we find

$$
\begin{aligned}
h_{6}^{(0)}(u) & =\pi J_{1}^{(9, \infty)}(u)+\frac{1}{\pi}\left[J_{1}^{(9, \infty)}(u) \int_{9}^{u} d t I_{1}^{(9, \infty)}(t)-I_{1}^{(9, \infty)}(u)\left(\int_{9}^{u} d t J_{1}^{(9, \infty)}(t)+5 \mathrm{Cl}_{2}\left(\frac{\pi}{3}\right)\right)\right] \\
& +i \pi I_{1}^{(9, \infty)}(u), \\
h_{7}^{(0)}(u) & =\pi J_{2}^{(9, \infty)}(u)+\frac{1}{\pi}\left[J_{2}^{(9, \infty)}(u) \int_{9}^{u} d t I_{1}^{(9, \infty)}(t)-I_{2}^{(9, \infty)}(u)\left(\int_{9}^{u} d t J_{1}^{(9, \infty)}(t)+5 \mathrm{Cl}_{2}\left(\frac{\pi}{3}\right)\right)\right] \\
& +i \pi I_{2}^{(9, \infty)}(u),
\end{aligned}
$$


recovering the correct results for the imaginary parts. Finally, we can write an alternative representation for the solution (3.26) as a dispersion relation

$$
\begin{aligned}
h_{6}^{(0)}(u)=\int_{9}^{\infty} \frac{d t}{t-u-i \varepsilon} I_{1}^{(9, \infty)}(t), \quad h_{7}^{(0)}(u) & =\frac{1}{\sqrt{3}} \mathrm{Cl}_{2}\left(\frac{\pi}{3}\right)+u\left(\frac{5}{6}+\sqrt{3} \mathrm{Cl}_{2}\left(\frac{\pi}{3}\right)\right) \\
& +u^{2} \int_{9}^{\infty} \frac{d t}{t^{2}(t-u-i \varepsilon)} I_{2}^{(9, \infty)}(t),
\end{aligned}
$$

where for $h_{7}^{(0)}(u)$ we have used a doubly subtracted dispersion relation and fixed the subtraction constants matching (3.29) to (3.26) for $u=0^{+}$and $u=1^{-}$.

The same exercise can be repeated at order $(d-4)$, obtaining relatively simple results, which we do not report here for brevity. We explicitly write down only the dispersive representation for the solution, as it will be used for the integration of the kite integral [26]

$$
\begin{gathered}
h_{6}^{(1)}(u)=\int_{9}^{\infty} \frac{d t}{t-u-i \varepsilon}\left(\frac{1}{4} I_{1}^{(9, \infty)}(t) \bar{l}(t)-\frac{\pi}{2} J_{1}^{(9, \infty)}(t)\right) \\
h_{7}^{(1)}(u)=\sqrt{3}\left[\frac{1}{6} \mathrm{Cl}_{2}\left(\frac{\pi}{3}\right) \ln (3)-\frac{1}{4} \mathrm{Ls}_{3}\left(\frac{2 \pi}{3}\right)-\frac{\pi^{3}}{72}\right] \\
+u\left[-\frac{5}{12}+\sqrt{3}\left(\frac{1}{2} \mathrm{Cl}_{2}\left(\frac{\pi}{3}\right) \ln (3)-\frac{3}{4} \operatorname{Ls}_{3}\left(\frac{2 \pi}{3}\right)+\frac{14}{27} \mathrm{Cl}_{2}\left(\frac{\pi}{3}\right)-\frac{\pi^{3}}{24}\right)\right] \\
+u^{2} \int_{9}^{\infty} \frac{d t}{t^{2}(t-u-i \varepsilon)}\left(\frac{1}{4} I_{2}^{(9, \infty)}(t) \bar{l}(t)-\frac{\pi}{2} J_{2}^{(9, \infty)}(t)+\frac{(t+3)^{2}}{6} I_{1}^{(9, \infty)}(t)\right),
\end{gathered}
$$

where, for $t>9$,

$$
\bar{l}(t)=2 \ln (t-1)+2 \ln (t-9)-\ln (t),
$$

and we introduced the generalization of the Clausen function

$$
\operatorname{Ls}_{n}(\theta)=-\int_{0}^{\theta} d y\left[\ln \left(2 \sin \left(\frac{y}{2}\right)\right)\right]^{n-1} .
$$

\subsection{The kite integral}

Equipped with formulas (3.29) (3.30) and (3.31), we are now ready to study the differential equation for the kite. $f_{8}(d ; u)$, Eq. (3.5), satisfies the linear first order differential equation

$$
\begin{aligned}
\frac{d}{d u} f_{8}(d ; u) & =(d-4)\left(\frac{1}{u-1}-\frac{1}{2 u}\right) f_{8}(d ; u)+\frac{(d-4)^{3}}{24}\left(1-\frac{8}{u-1}\right) h_{6}(d ; u) \\
& +\frac{(d-4)}{u-1}\left(-\frac{1}{8} f_{1}(d ; u)+2 f_{3}(d ; u)+f_{4}(d ; u)\right)+(d-4) \frac{1}{u} f_{5}(d ; u)
\end{aligned}
$$

where $f_{3}(d ; u), f_{4}(d ; u)$ and $f_{5}(d ; u)$ are simple master integrals espressible in terms of HPLs only, while $h_{6}(d ; u)$ is one of the sunrise integrals computed in the previous section. Expanding everything in Laurent series, we find for the first three orders, after fixing the boundary conditions

$$
f_{8}^{(0)}(u)=0, \quad f_{8}^{(1)}(u)=0, \quad f_{8}^{(2)}(u)=0 .
$$


The first non trivial order is the third one, which contains as inhomogeneous term the order zero of the sunrise graph. Using the dispersive solution for the latter (3.29), integrating explicitly over the simple denominator $(t-u)$ and fixing the boundary condition, we are left with an extremely compact result, again valid for $0<u<1$

$$
\begin{aligned}
f_{8}^{(3)}(u) & =\frac{1}{8} G(0,1,1, u)-\frac{1}{16} G(1,0,1, u)-\frac{\pi^{2}}{96} G(1, u) \\
& -\frac{1}{24} \int_{9}^{\infty} d t I_{1}^{(9, \infty)}(t)\left(1-\frac{8}{t-1}\right) G(t, u) .
\end{aligned}
$$

We stress that the solution contains only multiple-polylogarithms of maximum weight 3 together with integrals over elliptic integrals and logarithms. It is relatively straightforward to repeat the same exercise one order higher obtaining

$$
\begin{aligned}
f_{8}^{(4)}(u) & =\frac{\pi^{2}}{192}(G(0,1, u)-2 G(1,1, u))+\left(\frac{\zeta_{3}}{32}+\frac{\pi}{12} \mathrm{Cl}_{2}\left(\frac{\pi}{3}\right)\right) G(1, u)-\frac{3}{16} G(0,0,1,1, u) \\
& -\frac{1}{32} G(0,1,0,1, u)+\frac{3}{8} G(0,1,1,1, u)+\frac{1}{32} G(1,0,0,1, u)-\frac{1}{16} G(1,1,0,1, u) \\
& -\frac{1}{96} G(1, u) \int_{9}^{\infty} d t I_{1}^{(9, \infty)}(t) \bar{l}(t)+\frac{\pi}{48} \int_{9}^{\infty} d t J_{1}^{(9, \infty)}(t)\left(1-\frac{8}{t-1}\right) G(t, u) \\
& -\frac{1}{96} \int_{9}^{\infty} d t I_{1}^{(9, \infty)}(t)\left(1-\frac{8}{t-1}\right) \bar{l}(t)(G(t, u)-G(1, u)) \\
& +\frac{1}{48} \int_{9}^{\infty} d t I_{1}^{(9, \infty)}(t)\left(1-\frac{8}{t-1}\right)(G(0, t, u)-2 G(1, t, u)),
\end{aligned}
$$

where $\bar{l}(t)$ is defined in (3.32). Again the structure of the solution is very simple. It contains multiple-polylogarithms of weight 4 , together with integrals over elliptic integrals and polylogarithms of weight 2 . Our formalism allows to straightforwardly continue the results in any relevant region and, in particular, above the three massive particle threshold $u>9$. As exemplification, defining $v=(u-1) / 8$, we find for $u>9$

$$
\begin{aligned}
\frac{1}{\pi} \operatorname{Im} f_{8}^{(3)}(u) & =\frac{1}{16} G(0,-1 / 8, v)-\frac{1}{8} G(-1 / 8,0, v)-\frac{3}{8} \ln (2) G(-1 / 8, v) \\
& +\frac{1}{24} \int_{9}^{u} d t I_{1}^{(9, \infty)}(t)\left(1-\frac{8}{t-1}\right) .
\end{aligned}
$$

\section{Conclusions and outlook}

We have shown that the study of the imaginary part and of the corresponding dispersion relations of Feynman amplitudes within the differential equation method provides a powerful tool for handling their complexity, in particular when the solution lives outside the space of multiple polylogarithms. We have studied in detail the case of the two-loop massive sunrise graph and of the kite integral, relevant for the computation of the QED corrections to the electron propagator. We have derived compact results for the first two orders of the expansion in $(d-4)$, and showed how to continue them analytically in all relevant regions of the phase space. Our results are particularly well suited for numerical evaluation, as they involve at most one-fold integrals over elliptic integrals and multiple polylogarithms. An application of these techniques to more involved threeand four-point functions is currently underway. 


\section{References}

[1] K. Chetyrkin and F. Tkachov, Integration by Parts: The Algorithm to Calculate beta Functions in 4 Loops, Nucl.Phys. B192 (1981) 159-204.

[2] A. Kotikov, Differential equations method: New technique for massive Feynman diagrams calculation, Phys.Lett. B254 (1991) 158-164.

[3] E. Remiddi, Differential equations for Feynman graph amplitudes, Nuovo Cim. A110 (1997) 1435-1452, [hep-th/9711188].

[4] T. Gehrmann and E. Remiddi, Differential equations for two loop four point functions, Nucl.Phys. B580 (2000) 485-518, [hep-ph/9912329].

[5] E. Remiddi and J. Vermaseren, Harmonic polylogarithms, Int.J.Mod.Phys. A15 (2000) 725-754, [hep-ph/9905237].

[6] T. Gehrmann and E. Remiddi, Two loop master integrals for $\gamma^{*} \rightarrow 3$ jets: The Planar topologies, Nucl.Phys. B601 (2001) 248-286, [hep-ph/ 0008287 ].

[7] A. B. Goncharov, Multiple polylogarithms, cyclotomy and modular complexes, Math. Res. Lett. 5 (1998) 497-516, [1105.2076].

[8] A. B. Goncharov, Multiple polylogarithms and mixed Tate motives, math / 0103059.

[9] J. Ablinger, J. Bluemlein and C. Schneider, Analytic and Algorithmic Aspects of Generalized Harmonic Sums and Polylogarithms, J. Math. Phys. 54 (2013) 082301, [1302 . 0378].

[10] E. Panzer, Algorithms for the symbolic integration of hyperlogarithms with applications to Feynman integrals, Comput.Phys. Commun. 188 (2014) 148-166, [1403.3385].

[11] J. M. Henn, Multiloop integrals in dimensional regularization made simple, Phys.Rev.Lett. 110 (2013) 251601, [1304.1806].

[12] M. Argeri, S. Di Vita, P. Mastrolia, E. Mirabella, J. Schlenk, U. Schubert et al., Magnus and Dyson Series for Master Integrals, JHEP 1403 (2014) 082, [1401.2979].

[13] M. Caffo, H. Czyz, S. Laporta and E. Remiddi, The Master differential equations for the two loop sunrise selfmass amplitudes, Nuovo Cim. A111 (1998) 365-389, [hep-th/9805118].

[14] S. Laporta and E. Remiddi, Analytic treatment of the two loop equal mass sunrise graph, Nucl.Phys. B704 (2005) 349-386, [hep-ph/ 0406160$].$

[15] S. Bloch and P. Vanhove, The elliptic dilogarithm for the sunset graph, 1309.5865.

[16] E. Remiddi and L. Tancredi, Schouten identities for Feynman graph amplitudes; The Master Integrals for the two-loop massive sunrise graph, Nucl.Phys. B880 (2014) 343-377, [1311.3342].

[17] L. Adams, C. Bogner and S. Weinzierl, The two-loop sunrise graph with arbitrary masses, J.Math.Phys. 54 (2013) 052303, [1302.7004].

[18] L. Adams, C. Bogner and S. Weinzierl, The two-loop sunrise graph in two space-time dimensions with arbitrary masses in terms of elliptic dilogarithms, J.Math.Phys. 55 (2014) 102301, [1405. 5640 ].

[19] L. Adams, C. Bogner and S. Weinzierl, The two-loop sunrise integral around four space-time dimensions and generalisations of the Clausen and Glaisher functions towards the elliptic case, 1504.03255 .

[20] L. Adams, C. Bogner and S. Weinzierl, The iterated structure of the all-order result for the two-loop sunrise integral, 1512.05630. 
[21] S. Bloch, M. Kerr and P. Vanhove, Local mirror symmetry and the sunset Feynman integral, 1601.08181.

[22] L. Tancredi, Integration by parts identities in integer numbers of dimensions. A criterion for decoupling systems of differential equations, Nucl. Phys. B901 (2015) 282-317, [1509. 03330 ].

[23] R. N. Lee, Reducing differential equations for multiloop master integrals, JHEP 1504 (2015) 108, [1411.0911].

[24] J. Ablinger, A. Behring, J. Bl§mlein, A. De Freitas, A. von Manteuffel and C. Schneider, Calculating Three Loop Ladder and V-Topologies for Massive Operator Matrix Elements by Computer Algebra, Comput. Phys. Commun. 202 (2016) 33-112, [1509.08324].

[25] J. Ablinger, J. Bluemlein, A. de Freitas and C. Schneider, A toolbox to solve coupled systems of differential and difference equations, 1601.01856.

[26] E. Remiddi and L. Tancredi, Differential equations and dispersion relations for Feynman amplitudes. The two-loop massive sunrise and the kite integral, Nucl. Phys. B907 (2016) 400-444, [1602.01481].

[27] R. E. Cutkosky, Singularities and discontinuities of Feynman amplitudes, J. Math. Phys. 1 (1960) 429-433.

[28] M. J. G. Veltman, Unitarity and causality in a renormalizable field theory with unstable particles, Physica 29 (1963) 186-207.

[29] E. Remiddi, Dispersion Relations for Feynman Graphs, Helv. Phys. Acta 54 (1982) 364.

[30] O. V. Tarasov, Connection between Feynman integrals having different values of the space-time dimension, Phys. Rev. D54 (1996) 6479-6490, [hep-th/9606018].

[31] R. Lee, Space-time dimensionality D as complex variable: Calculating loop integrals using dimensional recurrence relation and analytical properties with respect to D, Nucl.Phys. $\mathbf{B 8 3 0}$ (2010) 474-492, [0911.0252]. 